OAl-PMH: http://www.indteca.com/ojs/index.php/Revista Scientific/oai

Artículo Original / Original Article

\title{
COVID-19 y comunicación de salud: Análisis de medios digitales ecuatorianos
}

\author{
Autor: Esteban Felipe Flores Romero \\ Universidad Internacional del Ecuador, UIDE \\ esfloresro@uide.edu.ec \\ Quito, Ecuador \\ https://orcid.org/0000-0002-8387-5886
}

\section{Resumen}

El trabajo analiza, mediante la Teoría de la Agenda Setting, la cobertura del brote de la COVID-19 en Ecuador, realizada por un Medio Digital Comunitario (MDCom) Wambra Ec y un Medio Digital Privado (MDPriv) GK. El objetivo es caracterizar las noticias en la primera fase del manejo de la pandemia por parte del estado ecuatoriano a partir del 29 de febrero al 25 de abril del año 2020, y contrastar con el trabajo de cada uno de los medios. Se realizó el análisis de contenido desde un abordaje cuantitativo y cualitativo. Las variables estudiadas fueron Tema, Título y Encuadre. El MDCom enfatizó su cobertura en la categoría Epidemiología, mientras que el MDPriv lo hizo en Educación en Salud. El MDCom no cubre Salud Mental y el MDPriv Derechos Sexuales y Reproductivos. El título de carácter informativo, al ser el mayor empleado por los medios, se observa que narra desde un inicio el contenido relacionado al tema, sea este epidemiológico, acceso a la salud o educación en salud. La agenda es empleada con los seguidores de diversas maneras y con los temas tratados, utilizando al medio como espacio de difusión para contarla.

Palabras clave: impacto de la comunicación; procesos de comunicación; medios electrónicos; medios comunitarios; flujo de noticias; covid-19.

Cómo citar este artículo:

Flores, E. (2021). COVID-19 y comunicación de salud: Análisis de medios digitales ecuatorianos. Revista Scientific, 6(19), 122-141, e-ISSN: 2542-2987. Recuperado de: https://doi.org/10.29394/Scientific.issn.2542-2987.2021.6.19.6.122-141

Fecha de Recepción: 09-09-2020
Fecha de Aceptación: 19-12-2020
Fecha de Publicación: 05-02-2021 
OAI-PMH: http://www.indteca.com/ojs/index.php/Revista Scientific/oai

Artículo Original / Original Article

\title{
COVID-19 and health communication: Analysis of Ecuadorian digital media
}

\begin{abstract}
The work analyzes, through the Agenda Setting Theory, the coverage of the COVID-19 outbreak in Ecuador, carried out by a Community Digital Media (MDCom) Wambra Ec and a Private Digital Media (MDPriv) GK. The objective is to characterize the news in the first phase of the handling of the pandemic by the Ecuadorian state from February 29 to April 25, 2020, and contrast with the work of each of the media. The content analysis was carried out from a quantitative and qualitative approach. The variables studied were Topic, Title and Frame. The MDCom emphasized its coverage in the Epidemiology category, while the MDPriv did so in Health Education. The MDCom does not cover Mental Health and the MDPriv Sexual and Reproductive Rights. The informative title, being the largest used by the media, is observed from the beginning narrates the content related to the topic, be it epidemiological, access to health or health education. The agenda is used with the followers in various ways and with the topics discussed, using the medium as a space for dissemination to tell it.
\end{abstract}

Keywords: impact of communication; communication processes; electronic media; community media; news flow; covid-19.

How to cite this article:

Flores, E. (2021). COVID-19 and health communication: Analysis of Ecuadorian digital media. Revista Scientific, 6(19), 122-141, e-ISSN: 2542-2987. Recovered from: https://doi.org/10.29394/Scientific.issn.2542-2987.2021.6.19.6.122-141

Date Received:

09-09-2020
Date Acceptance:

19-12-2020
Date Publication:

05-02-2021 


\section{Introducción}

A finales de diciembre del año 2019, como indica Acosta, Escobar, Bernaola, Alfaro, Taype, Marcos y Amado (2020): desde la ciudad de Wuhan, China, se notificó a la comunidad internacional de un brote epidémico ocasionado por el virus SARS-CoV-2. Tras el primer caso diagnosticado en Ecuador, el febrero 29 del año 2020, y la declaración de pandemia por parte de la Organización Mundial de la Salud (OMS, 2020): el 11 de marzo del año 2020, el gobierno ecuatoriano declaró el Estado de Emergencia Sanitaria el 12 de marzo, y proclamó el Estado de Excepción el 16 de marzo del mismo año. Esta situación jurídica permitió al Ecuador declarar medidas para controlar la pandemia definidas en el Decreto Presidencial N0 1017 del 2020.

Según el Ministerio de Salud Pública (MSP, 2020): hasta noviembre del año 2020, la enfermedad causada por este virus, COVID-19, causó en Ecuador más de 185 mil casos y más de 8,8 mil fallecidos. A los 57 días del 25 de abril del año 2020, desde primer caso diagnosticado, el gobierno ecuatoriano cambió la estrategia del confinamiento por el distanciamiento social para la contención de la pandemia.

Desde el debut de esta enfermedad, los medios de comunicación incorporaron la pandemia como eje de su cobertura. Para Lázaro-Rodríguez y Herrera-Viedma (2020): el seguimiento del progreso de la pandemia, así como los temas que giran a su alrededor, han marcado tendencia en la comunicación, tanto en las redes sociales, así como en los medios tradicionales y medios digitales.

Los medios digitales aparecieron como un espacio para que los medios tradicionales expongan su contenido desde una nueva plataforma de difusión, acorde con esto, Rivera (2013a): expone que "a comienzos del 1995, uno de los grandes medios The Washington Post, dio sus primeros pasos en Internet" (pág. 112); empezando de esta forma una carrera por generar contenido y estrategias para mantener a sus audiencias en este canal de comunicación. 
Los medios digitales en Ecuador, para Rivera (2013b): en un inicio, contaban lo que pasaba en el país para quienes quisieran informarse en el exterior. Según Morán (2015a): en abril del año 2015 existen 60 medios nativos digitales, es decir medios que trabajan desde lo digital y no como adaptación.

Los medios digitales ya fueron descritos en su momento por Jenkins, Ford y Green (2015): como nuevos canales de producción, nuevos contenidos y nuevas relaciones entre producción y audiencia. Así mismo, Scolari (2012a): indica que estos nuevos medios alteraron la esfera de la comunicación, teniendo ahora a la audiencia como guía de construcción del contenido, es decir son colaboradores. De esta forma estos medios llegan a tener una identidad propia y mostrar un pensamiento con el movimiento de contenido entre los usuarios.

En esa misma línea, Morán (2015b): describe a los medios digitales en Ecuador con una necesidad de tener una estrategia de difusión, más no como un espacio dónde sólo replican noticias de otros portales 0 de sus transmisiones por televisión, radio o prensa. Por otro lado, el derecho al acceso a los medios de comunicación en igualdad de condiciones, contemplado en la Constitución del Ecuador y manejado por la Ley Orgánica de Comunicación (LOC, 2013a): describe tres formas de administración de medios de comunicación: públicos, privados y comunitarios.

Para este caso nos interesa los medios privados y comunitarios. Los medios comunitarios son definidos por la Ley Orgánica de Comunicación (LOC, 2013b): como "[...] aquellos cuya propiedad, administración y dirección corresponden a los movimientos y organizaciones sociales, colectivos [...]" (pág. 44). La diferencia de los medios comunitarios con los medios privados, en términos generales, es que los medios comunitarios buscan un fin social, de ayuda comunitaria o promover a minorías que han sido históricamente relegadas del acceso a medios de comunicación, mientras que los medios 
privados tienen como fin el lucro de quienes manejan el medio.

Conforme a McCombs y Evatt (1995): Ios medios "al prestar atención a ciertos aspectos, a expensas de otros, y al sugerir ciertas soluciones o respuestas en lugar de otras, los mensajes de los medios de difusión influyen en algo más que en los temas [...]" (pág. 22). La Teoría de la Agenda Setting se centra en analizar el efecto que ejercen los medios sobre las audiencias con respecto a temas definidos y establecidos por el interés de los medios. De igual manera la Teoría del Framing, entendida como el tratamiento de los medios con los hechos presentados, de acuerdo con Armentia, Marín, Rodríguez y Marauri (2019a): sirve para analizar lo encontrado en el análisis.

En Ecuador se han producido un sinfín de noticias en el eje de la salud por la pandemia; sin embargo, no existen estudios académicos que usen la Teoría de la Agenda Setting y la Teoría del Framing para analizar las noticias que abordan los medios digitales ni las diferencias o similitudes en los contenidos entre medios digitales privados y comunitarios, en el marco de la COVID-19.

Con estos antecedentes, el objetivo de esta investigación es caracterizar el abordaje temático de los medios digitales en la pandemia por la COVID-19 en Ecuador, durante el periodo de confinamiento entre el 29 de febrero al 25 de abril del año 2020, bajo el paradigma de la Teoría de Agenda Setting en el medio digital privado y digital comunitario.

\section{Metodología (materiales y métodos)}

Este estudio se basa en un diseño mixto de tipo cuantitativo y cualitativo. El período de tiempo para la recolección de noticias fue desde el 29 de febrero hasta el 25 de abril del año 2020, un día después del anuncio del cambio a la etapa de distanciamiento.

La selección del medio digital se realizó a partir de los siguientes criterios de inclusión: presencia en el periodismo digital ecuatoriano al menos 
12 meses antes y durante la pandemia, tener plataforma web propia para su difusión, que no sean un medio regional ni portal de investigación o especializado y que el formato empleado sea escrito. Se revisaron nueve medios digitales, de los cuales seis son privados y tres comunitarios. Los medios digitales que cumplieron con los criterios de inclusión fueron el Medio Digital Comunitario (MDCom), Wambra Ec; y el Medio Digita Privado (MDPriv), GK.

El material de estudio se centra en las noticias publicadas en los portales web del MDCom y del MDPriv. Las noticias seleccionadas estuvieron relacionadas a la COVID-19. El sondeo inicial se realizó con la palabra motivo del estudio en los buscadores de los portales web de los medios indicados. En una segunda instancia se realizó una búsqueda ampliada por las secciones en su página web de contenido relacionado con el tema de búsqueda, obteniendo (84) noticias, (55) del MDPriv y (29) del MDCom. De este universo se usaron las noticias que tratan la temática de salud. El número de noticias empleadas fue de (29) del MDPriv y (17) del MDCom.

El trabajo del MDCom se centra en crear oportunidades para narrar historias enfocadas en los derechos humanos y el feminismo, así como esparcir el poder de la comunicación. Mientras que el servicio del MDPriv es contextualizar los hechos que ocurren en el Ecuador y en el mundo para poder crear espacios de discusión y conocimiento entre las partes involucradas.

\subsection{Abordaje cuantitativo}

La primera variable descrita en el abordaje cuantitativo es el Tema. Esta variable fue categorizada, siguiendo a Ordorika (2020); y Tizón (2020): en a). Salud Mental (relación entre el mundo psico-emocional y el contexto sociocultural de las personas con respecto a la pandemia); b). Educación en Salud (explicación del funcionamiento de los temas de salud de la pandemia, el desarrollo del virus, medidas de contagio, la promoción, el mantenimiento o 
la restauración de la salud); y c). Acceso a la Salud (derecho a la salud por parte de la ciudadanía y las situaciones que puede vivir el personal sanitario que afecte el acceso a salud y la prevención de enfermedades).

Además de estos tres temas mencionados se añadieron las categorías de: d). Epidemiología definida acorde a Cardona (2016): como el conteo actual de personas contagiadas, fallecidos y recuperados por COVID-19; y e). Derechos Sexuales y Reproductivos, como lo conciben Massó y Triviño (2020): motivado en las vulneraciones con respecto a este tema, generada durante la pandemia.

La segunda variable es el Título que tiene la noticia, dividida en cuatro categorías explicadas por Armentia, Marín, Rodríguez y Marauri (2019b): a). Informativo, con estructura de sujeto + verbo + predicado con nociones generales al lector del contenido; b). Interrogativo, se plantea una duda que se responde en el texto; c). Numérico, se da al lector una lista y se expone la causa de una consecuencia; y d). Demostrativo, que señala información precisa al lector acerca de un tema, además del uso de un pronombre demostrativo.

De acuerdo con Canel (1999): la tercera variable es el Encuadre de la noticia, el enfoque responde al contexto que da el medio a la noticia presentada, al trabajo como medio con el que se le podría filiar. Para Armentia, Marín, Rodríguez y Marauri (2019c): las categorías fueron: a). Diagnóstico (indagación de un evento); b). Consecuencia (los hechos desencadenados de un hecho); c). Responsabilidad (responsabilidades en un hecho); y d). Soluciones (sugerencias a un hecho).

La descripción de estas variables categóricas se realiza mediante frecuencias absolutas y relativas; además, se analiza la asociación entre el tema con el título, medio y tono, así como título y tono, a través de distribuciones conjuntas, en el software estadístico informático SPSS en su versión nro. 24. 


\subsection{Abordaje cualitativo}

La muestra del componente cualitativo se construyó con la transcripción y codificación de las noticias que cumplieron los criterios de inclusión, fueron analizadas bajo el método de Análisis de Contenido mediante la selección de frases significativas en las cuatro variables consideradas (medio, tema, título y tono), con respecto a lo antes mencionado, Porta y Silva (2003): manifiestan que es a partir de criterios de concordancia y discrepancia entre los medios digitales participantes. El control de calidad de los datos generados fue realizado mediante la triangulación metodológica con un experto en Epidemiología y Salud Pública y dos en Comunicación.

\section{Análisis y resultados}

\subsection{Resultados abordaje cuantitativo}

En la variable Tema, en el MDCom se observan 11 noticias $(64,7 \%)$ que abordan la Epidemiología, mientras que el MDPriv tiene 13 publicaciones $(44,8 \%)$ relacionadas con Acceso a la Salud. Los titulares empleados revelan que el MDCom hace uso en un 100,0\% (17) del tipo informativo y el MDPriv hace uso en un 75,9\% (22) de esta opción. En el tono de las noticias el MDCom hace uso del tono Consecuencias en 13 (76,5\%) ocasiones, por su parte el MDPriv hace uso del tono diagnóstico en un $51,7 \%$ (15). El MDPriv hace uso de todos los tonos, mientras que el MDCom no usa Responsabilidad y Soluciones.

El gráfico 1, muestra la relación entre el Tema y el Tono de los artículos, de manera global y por cada medio. El gráfico $1 \mathrm{~A}$ muestra que en la categoría Diagnóstico el Acceso a la Salud se encontró en 10 de 19 (52,6\%) noticias. En la categoría Consecuencias el tema de Epidemiología se encontró en 13 de $21(61,9 \%)$ noticias. En las categorías Responsabilidad y Soluciones se encontraron noticias de Acceso a la Salud (1/1; 100,0\%) y Educación en Salud $(3 / 5 ; 60,0 \%)$, respectivamente. 
En el caso del MDCom, en el gráfico 1B, se observa que en la categoría Diagnóstico el Acceso a la salud se encontró en 2 de $4(50,0 \%)$ y en la categoría Consecuencias las noticias relacionadas con Epidemiología se encontraron en 11 de $13(84,6 \%)$ registros. Las categorías temáticas no fueron encontradas en los tonos Responsabilidad y Soluciones. En el MDPriv, se representa en el gráfico $1 \mathrm{C}$, el tema Acceso a la Salud se encontró en las categorías Diagnóstico (8/15; 53,3\%), Consecuencias (3/8; 37,5\%) y Responsabilidad (1/1; 100,0\%). El tema Educación en Salud se encontró en la categoría Soluciones $(3 / 5 ; 60,0 \%)$.

Gráfico 1. El Tema y el Tono del artículo (1A, 1B, 1C).
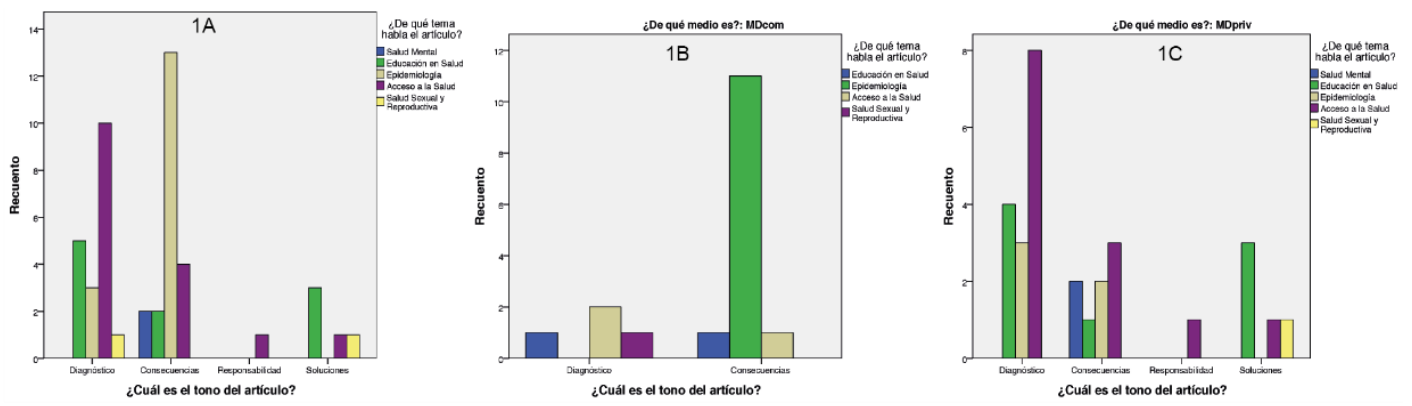

Fuente: El Autor (2020).

El gráfico 2, muestra la relación que existe entre el Tema y el Título de los artículos, de manera global y por cada medio. El gráfico $2 \mathrm{~A}$, muestra que en la categoría Informativo la Epidemiología se encontró en 14 de 39 (35,9\%) noticias. En la categoría Interrogativo los temas Acceso a la Salud y Educación en Salud se encontró de manera similar $(1 / 2 ; 50,0 \%)$ en las noticias. En las categorías Numérico y Demostrativo se encontraron noticias de Epidemiología $(2 / 3 ; 66,7 \%)$ y Acceso a la Salud $(2 / 3 ; 66,7 \%)$, respectivamente.

En el caso del MDCom, se observa en el gráfico 2B, que en la categoría Informativo la Epidemiología se encontró en 11 de 17 (64,7\%) ocasiones. En el MDPriv, como se expone en el gráfico $2 \mathrm{C}$, con el tema Acceso a la Salud se encontró en las categorías Informativo (9/22; 40,9\%) y Demostrativo (2/2; 
$100,0 \%)$. El tema Educación en Salud se encontró en la categoría Interrogativo (3/5; $60,0 \%)$ y el tema Epidemiología en la categoría Numérico (2/3; 66,7\%).

Gráfico 2. El Tema y el Título del artículo (2A, 2B, 2C).
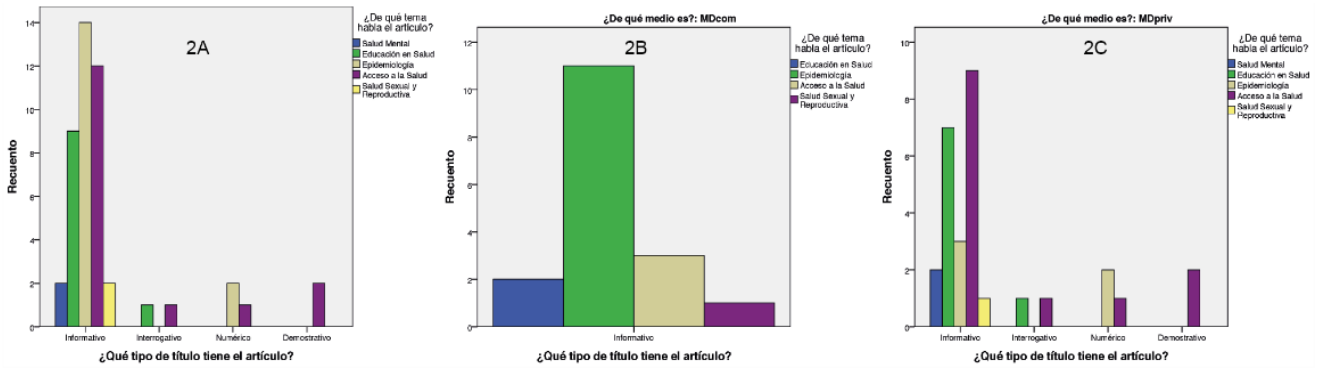

Fuente: El Autores (2020).

El gráfico 3, muestra la relación que existe entre el Título y el Tono de los artículos, de manera global y por cada medio. El gráfico 3A, demuestra que el Título Informativo tiene mayor presencia en las categorías Diagnóstico (15/19; 78,9\%), Consecuencias (19/21; 90,5\%), Responsabilidad (1/1; $100,0 \%)$ y Soluciones $(4 / 5 ; 80,0 \%)$.

En el caso del MDCom, en el gráfico 3B, se evidencia que el titular Informativo está presente sólo en las categorías Diagnóstico $(4 / 4 ; 100,0 \%)$ y Consecuencias (13/13; 100,0\%). En el MDPriv, como se exhibe en el gráfico $3 \mathrm{C}$, el titular Informativo se encontró en las categorías Diagnóstico (11/15; $73,3 \%)$, Consecuencias $(6 / 8 ; 75,0 \%)$, Responsabilidad $(1 / 1 ; 100,0 \%)$ y Soluciones $(4 / 5 ; 80,0 \%)$.

Gráfico 3. El Tema y el Título del artículo (3A, 3B, 3C).
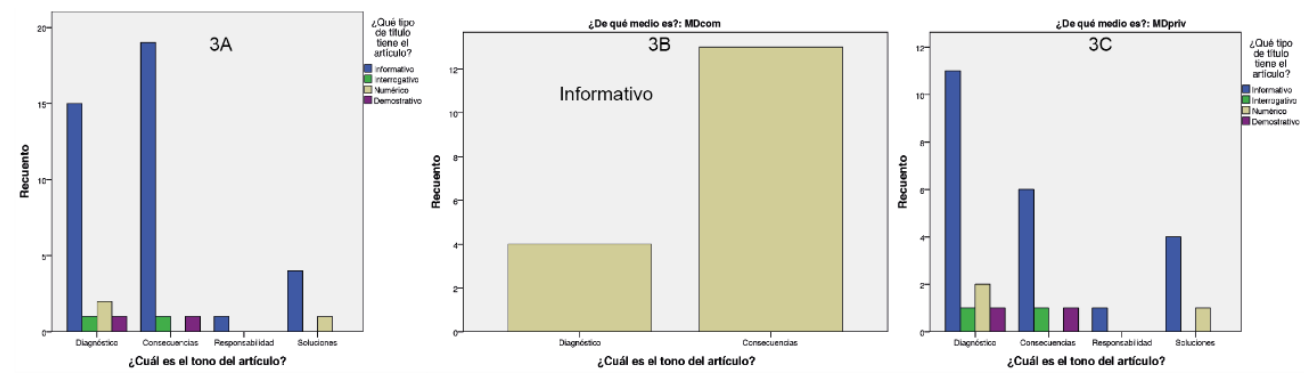

Fuente: El Autor (2020). 


\subsection{Resultados del abordaje cualitativo}

En las noticias analizadas del MDCom se observa al titular Informativo junto al tema Epidemiología en reiteradas ocasiones. Esta distribución describe lo que el lector va a encontrar en su contenido, empezando con el tema coronavirus e inmediatamente con un resumen junto al día correspondiente, las fuentes que se usan y el orden. Como parte del encabezado se encontró en varias ocasiones el uso de hashtags para identificar el tema y hacer hincapié en el medio que redacta la noticia.

Desde la transcripción con código MDCom11 a la MDCom14, se encuentra un párrafo explicativo inicial, donde se hace referencia a la cantidad de pruebas COVID-19 que se tenían en el país, tomándose en cuenta que no eran suficientes, por lo tanto, las cifras presentadas por las autoridades no eran acordes a la situación del país (MDCom11 - MDCom14: las cifras oficiales de personas contagiadas y fallecidas por COVID-19 en Ecuador corresponden solo una parte de la realidad).

EI MDPriv hace uso igualmente del titular Informativo en sus temas. En un ejemplo que ilustra la estructura se puede ver que describe inmediatamente el contenido (MDPriv14: La carrera contra el tiempo para aumentar las pruebas de coronavirus en América Latina). De igual manera al ser un Tema de Acceso a la Salud usa frases que apoyan el tema central (MDPriv14: el número de personas a las que se les hace la prueba es muy bajo, no se tiene una fotografía completa del problema). En este caso, además de la idea central, el medio presenta una sección que permite al lector entender las pruebas y por qué se vive la situación del diagnóstico del coronavirus en América Latina.

En el Título de carácter Informativo, al ser el mayor empleado por los medios, se observa que narra desde un inicio el contenido relacionado al tema, sea este epidemiológico, acceso a la salud o educación en salud. El MDCom menciona desde un inicio quién y qué desarrolla sus actividades durante esta pandemia (MDCom1: ser enfermera rural en épocas de COVID-19); en 
cambio, el MDPriv dice qué pasa con un objetivo planteado en un territorio específico (MDPriv14: la carrera contra el tiempo para aumentar las pruebas de coronavirus en América Latina).

El tono empleado mayormente por el MDCom (Consecuencias) se distingue en frases que sintetizan el texto (MDCom1: han tomado la decisión de adquirir trajes especiales para disminuir el riego), que junto a la declaración de la acción de dividirse los guantes con una compañera para poder aprovechar la poca cantidad de insumos que tienen respalda la identidad del medio con los temas.

En el Tono de Consecuencias en el MDPriv tiene temas como Acceso a la Salud o Educación en Salud, este último, por ejemplo, donde habla de los efectos que tiene el coronavirus en los niños y que desde el titular se plantea como interrogación. El texto se desarrolla respondiendo a la inquietud, haciendo referencia a estudios o entidades que muestran respuestas. Uno de los ejemplos menciona que no había certeza acerca de cómo se comportaba el virus en niños. Por otro lado, abordaron aspectos en recién nacidos (MDPriv3: los primeros estudios de casos en Wuhan descartaron que las madres transmitan el virus a sus hijos recién nacidos).

El Tema de Acceso a la Salud y el Tono de Diagnóstico en ambos medios tienen frases que afirman estas características dentro de los textos, apoyando los objetivos de cada uno de estos que tienen en su trabajo como medios. En el MDCom hay frases que apuntan a las historias que no son contadas cotidianamente (MDCom1: los profesionales de salud recorren la comunidad diariamente); mientras que el MDPriv crea un contexto de la crisis a nivel regional (MDPriv14: varios países de América Latina están reaccionando ante sus falencias en la capacidad de diagnóstico).

Los temas de Acceso a la Salud y Derecho Sexuales y Reproductivos en el MDCom se leen con un Tono de Consecuencia (MDCom3: lo que quiero decirles como paciente autoinmune), mostrando el relato de pasar aislada, 
pero que de igual forma tiene que salir con extremo cuidado. Las pastillas que ella necesita son de igual relevancia en la narración, ella usa Hidroxicloroquina, que en la fecha de publicación de la nota, 31 de marzo del año 2020, se discutió sus efectos contra la COVID-19. Dada la cantidad de información circulando en internet llevó a que la gente compre el medicamento disponible en las farmacias (MDCom3: cual grageas de fresa). Como parte del encuadre que tiene la nota finaliza (MDCom3: la cuarentena por el COVID-19 no es igual para todos y todas), haciendo énfasis en que cada uno debe cuidarse por la pandemia.

Por último, al estar el título fuertemente ligado con el tono de consecuencia, se ven ejemplos en ambos medios que comprueban esta situación. Por parte del MDCom se ve como usan la estructura de resúmenes por días, mostrando las consecuencias que tiene la pandemia en el país. El MDPriv tiene la misma situación, con titulares que enfatizan las consecuencias de la pandemia (MDPriv25: si buscas el cuerpo de un familiar fallecido en la emergencia del COVID-19, súmalo a esta lista).

\subsection{Discusión}

Los medios digitales, al ser quienes llevan la discusión a la sociedad, los distintos temas y puntos de vista, han tenido que establecer distintas agendas al momento de hablar de la COVID-19. Cada medio, dependiendo de los canales de comunicación que mantenga con sus comunidades, genera el contenido y encuadra los distintos temas acordes a su estilo. MDCom y MDPriv tienen temas que fueron cubiertos en el mayor número de ocasiones en las áreas de epidemiología y educación en salud respectivamente. En el caso del MDCom el tema que ha quedado sin interés es el de Salud Mental, mientras que para el MDPriv es el de Derechos Sexuales y Reproductivos.

En tal sentido, Lubens (2014): plantea que las funciones de los medios en un brote epidémico son: las de dar información concreta a la ciudadanía, 
así como a los profesionales de la salud, quienes toman las decisiones y el de emplear el papel de veedores del accionar de las instituciones que manejan dicho brote.

La principal diferencia se encuentra en el enfoque y tratamiento que se le da a la información de cada tema por medio digital, así como el espacio en el que se desenvuelve cada uno. Las plataformas web y el canal de comunicación de cada medio, Rivera (2013c): establece que no llega a ser un espacio donde transcriben contenido de un sitio a este, tal y como se hacía en el año 1999, sino juega un papel importante por cómo es presentada la información.

En el período de tiempo analizado la información manejada en el país, en un inicio, fue presentada desde el exterior y a partir del primer caso de COVID-19 se empezó a tener publicaciones con información generada desde el país. En el caso del MDCom ese fue su principal contenido, informar las consecuencias de la pandemia y el estado del Acceso a Salud de la población, con la guía de su trabajo como medio, desperdigando el poder y relatando hechos que no son contados habitualmente. Tal como es el caso de una paciente con enfermedad autoinmune o de medidas en la población que van en contra de los derechos de Lesbianas, Gais, Bisexuales, Transexuales, Intersexuales y Queer (LGBTIQ+).

El MDPriv por su lado se enfocó en el Acceso a la Salud, no solo del país, sino que contextualizó la situación con el resto de Latinoamérica. El enfoque de trabajo para este medio es la empatía, el contexto y aterrizar los sucesos a la población, por esta razón la Educación en Salud no dejó de ser un tema importante para su cobertura.

Con estos hechos tratados por los medios se observa que un mismo tema es tratado diferente por varios medios, así como expone Aruguete (2009): más aún cuando entran variables como Título y Tono empleados. El contenido de MDCom11 al MDCom14 muestran un punto de vista de la 
situación del país, recalcando que el enfoque que tiene este medio es dirigido, justamente, hacia las minorías sociales, que en este son quienes no pudieron acceder a una prueba COVID-19. Mientras que el MDPriv hace hincapié en la estructura del sistema de salud y qué puntos son los más vulnerables dentro del mismo, de esta manera buscando que se fortalezca, mostrando que el encuadre de la información, así como su agenda se centró en el contexto de la situación, no solo nacional sino regional.

Ahora bien, esto se refleja justamente en el medio y en su comunidad, Albarello (2020): mencionó que "las comunidades de fans [...] traen consigo muchas capacidades [...]" (pág. 173); en este sentido, Scolari (2012b): manifiesta que los seguidores ya no son solo personas externas al medio, sino son parte del medio mismo, este consigue imponer sus ideas y trabajo en sus seguidores. Imponer las ideas hoy en día conlleva mantener el enfoque de trabajo del medio siempre presente, por lo que el medio desde este aspecto viene con su agenda marcada desde su concepción. La agenda es empleada en y con los seguidores de diversas maneras junto a los temas tratados, así como lo describe Benítez y Hidalgo (2020): utilizando al medio como espacio de difusión para visualizar la agenda a través de los temas tratados y las noticias generadas.

\section{Conclusiones}

El MDCom, dentro de sus temáticas tratadas, tiene mayor disposición por los titulares Informativos. Así como el tono de Consecuencias, exponiendo el resultado del manejo de la epidemia por parte del gobierno, y sus efectos en los distintos sectores de la sociedad.

EI MDPriv, emplea el titular Informativo en su mayoría. Por otro lado, el tono es el de Diagnóstico, presentando la situación actual del país para quien los lea, así como las medidas tomadas para mitigar la pandemia por parte del gobierno. 
La hipótesis de este trabajo plantea que la atención a ciertos temas específicos en lugar de otros, en un medio digital privado y uno digital comunitario, va acorde con el enfoque de trabajo de cada uno, que en base a los resultados presentados se evidencia lo mencionado.

De igual manera el objetivo es cumplido en la medida de que las variables y categorías abordadas demuestran el interés de los medios por hablar de ciertos temas sobre otros en base a su trabajo; sin embargo, habría que desarrollar las mismas dudas con una diferencia de mayores temáticas y no solamente en el eje de salud.

\section{Referencias}

Acosta, G., Escobar, G., Bernaola, G., Alfaro, J., Taype, W., Marcos, C., \& Amado, J. (2020). Caracterización de Pacientes con COVID-19 grave Atendidos en un Hospital de Referencia Nacional del Perú. Revista Peruana de Medicina Experimental y Salud Pública, 37(2), 253-258, eISSN: 1726-4642. Recuperado de:

https://doi.org/10.17843/rpmesp.2020.372.5437

Albarello, F. (2020). Henry Jenkins: "Las comunidades de fans se están volviendo políticas”. Austral Comunicación, 9(1), 171-184, e-ISSN: 2313-9137. Recuperado de:

https://dialnet.unirioja.es/servlet/articulo?codigo $=7502829$

Armentia, J., Marín, F., Rodríguez, M., \& Marauri, I. (2019a,b,c). De qué habla la prensa digital cuando habla de nutrición. Un análisis de elpais.com y lavanguardia.com durante 2017. Doxa Comunicación: Revista interdisciplinar de estudios de comunicación y ciencias sociales, (29), 19-41, e-ISSN: 2386-3978. Recuperado de:

http://hdl.handle.net/10637/10733

Aruguete, N. (2009). Estableciendo la agenda. Los orígenes y la evolución de la teoría de la Agenda Setting. Ecos de la comunicación, 2(2), 11- 
38, e-ISSN: 1852-0464. Recuperado de:

https://repositorio.uca.edu.ar/handle/123456789/7119

Benítez, M., \& Hidalgo, P. (2020). Comportamientos generacionales y contenidos informativos que construyen el mundo de las Fake News en Ecuador. Revista Scientific, 5(16), 208-229, e-ISSN: 25422987. Recuperado de: https://doi.org/10.29394/Scientific.issn.25422987.2020.5.16.11.208-229

Canel, M. (1999). EI País, ABC y EI Mundo: Tres manchetas, tres enfoques de las noticias. Zer: Revista de estudios de comunicación = Komunikazio ikasketen aldizkaria, (6), 1-11, e-ISSN: 1137-1102 Recuperado de:

https://dialnet.unirioja.es/servlet/articulo?codigo $=826304$

Cardona, J. (2016). Determinantes y Determinación Social de la Salud como confluencia de la salud pública, la epidemiología y la clínica. Archivos de Medicina (Manizales), 16(1), 183-191, e-ISSN: 2339-3874. Recuperado de: https://doi.org/10.30554/archmed.16.1.1090.2016 Jenkins, H., Ford, S., \& Green, J. (2015). Cultura Transmedia: La creación de contenido y valor en una cultura en red. Primera Edición, ISBN: 978-84-9784-845-9. Barcelona, España: Editorial Gedisa.

LOC (2013a,b). Ley Orgánica de Comunicación. Ley 0, Registro Oficial Suplemento 22 de 25 junio, Ultima modificación: 20 de febrero de 2019, Estado: Reformado. Ecuador: Asamblea Nacional.

Lubens, P. (2014). Journalists and Public Health Professionals: Challenges of a Symbiotic Relationship. Disaster Medicine and Public Health Preparedness, 9(1), 59-63, e-ISSN: 1935-7893. Retrieved from: https://doi.org/10.1017/dmp.2014.127

Lázaro-Rodríguez, P., \& Herrera-Viedma, E. (2020). Noticias sobre Covid-19 y 2019-nCoV en medios de comunicación de España: el papel de los medios digitales en tiempos de confinamiento. El profesional de 
la información, 29(3), 1-11, e-ISSN: 1699-2407. Recuperado de: https://doi.org/10.3145/epi.2020.may.02

Massó, E., \& Triviño, R. (2020). Parto y aborto en tiempos de coronavirus: el impacto de la pandemia en los derechos sexuales $y$ reproductivos. Enrahonar. An international journal of theoretical and practical reason, 65, 117-130, e-ISSN: 2014-881X. Recuperado de: https://doi.org/10.5565/rev/enrahonar.1305

McCombs, M., \& Evatt, D. (1995). Los temas y los aspectos: explorando una nueva dimensión de la agenda setting. REV: Comunicación y Sociedad, 8(1), 7-32, e-ISSN: 0214-0039. Recuperado de: https://dadun.unav.edu/handle/10171/8401

Morán, S. (2015a,b). El auge de los medios nativos digitales en Ecuador. Ecuador: Fundación Andina de Observación y Estudio Social de los Medios - Fundamedios.

MSP (2020). Situación Nacional por COVID-19. Infografía N²69. Ecuador: Ministerio de Salud Pública.

OMS (2020). La OMS caracteriza a COVID-19 como una pandemia. Ginebra, Suiza: Organización Panamericana de la Salud; Organización Mundial de la Salud.

Ordorika, I. (2020). Pandemia y educación superior. RESU: Revista de la Educación Superior, 49(194), 1-8, e-ISSN: 2395-9037. Recuperado de: http://189.254.1.230/ojs/index.php/resu/article/view/1120

Porta, L., \& Silva, M. (2003). La investigación cualitativa: El Análisis de Contenido en la investigación educativa. Anuario Digital de Investigación Educativa, (14), 388-406, e-ISSN: 2618-3862. Recuperado de:

http://revistas.bibdigital.uccor.edu.ar/index.php/adiv/article/view/3301

Rivera, J. (2013a,b,c). Medios digitales en Ecuador, cuántos son y qué hacen. Chasqui: Revista Latinoamericana de Comunicación, (122), 
111-117, e-ISSN: 1390-1079. Recuperado de:

http://hdl.handle.net/10469/13303

Scolari, C. (2012a,b). Comunicación digital. Recuerdos del futuro. El profesional de la información, 21(4), 337-340, e-ISSN: 1386-6710. Recuperado de: https://doi.org/10.3145/epi.2012.jul.01

Tizón, J. (2020). Salud emocional en tiempos de pandemia. 1ra Edición, ISBN: 978-84-254-4606-1. Barcelona, España: Herder Editorial, S.L. 


\section{Esteban Felipe Flores Romero \\ e-mail: esfloresro@uide.edu.ec}

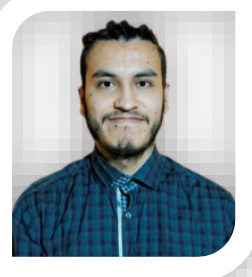

Nacido en Quito, Ecuador, el 15 de agosto del año 1997. Licenciado en Comunicación Organizacional y Relaciones Públicas por la Universidad Internacional del Ecuador (UIDE); con experiencia en creación de campañas para difusión y posterior implementación de contenido cultural; además de la creación de contenido para la divulgación de proyectos sociales y de salud; he trabajado en el área de comunicación con los proyectos musicales quiteños Celtic Trip (2018); Korioto (2020); y Sumo Semblante (2020), en el lanzamiento de su material; y soy parte del equipo de difusión de la productora musical Warax Arte de Quito, Ecuador.

El contenido de este manuscrito se difunde bajo una Licencia de Creative Commons ReconocimientoNoComercial-Compartirlgual 4.0 Internacional 